\title{
Effects of solid-state fermentation on the nutritional components and antioxidant properties from quinoa
}

\author{
L. N. Xu' ${ }^{1}$ S. Guo ${ }^{2}$, Sheng Wan Zhang ${ }^{1 *}$ \\ ${ }^{1}$ School of Life Sciences, Shanxi University, Taiyuan 030006, China, ${ }^{2}$ Shanxi Academy of Agricultural Sciences, Institute of Edible Fungi, \\ Taiyuan 030031 , China
}

\section{A B S T R A C T}

This study aimed to evaluate the effects of solid-state fermentation (SSF) ( $25^{\circ} \mathrm{C}, 35$ days) with three filamentous fungi (He/vella lacunosa X1, Agaricus bisporus AS2796 and Fomitiporia yanbeiensis G1) on the nutrient substance and antioxidant properties of quinoa. As a result, it showed that the nutritional components in fermented - quinoa varied with the starter strains. Among the three starter fungi, AS2796 gave the highest protein contents $(28.46 \mathrm{~g} / 100 \mathrm{~g})$, which was 2.34 times higher than control (unfermented quinoa); $F$. G1 gave the lowest values of soluble starch and crude fat $(18.46 \mathrm{~g} / 100 \mathrm{~g}$ and $3.31 \mathrm{~g} / 100 \mathrm{~g})$, which were $35.2 \%$ and $58.5 \%$ lower than that of control, respectively; and $H$. X1 gave the highest content of reducing sugar $(5.62 \mathrm{~g} / 100 \mathrm{~g})$, which was 5.50 times higher than that of control. The total phenolics of quinoa fermented by AS2796 reached its maximum value $(1.38 \mathrm{mg} / \mathrm{g}$, 35 days $)$, which was 1.97 holds higher than control. According to antioxidant test in vitro of ethanolic extracts of fermented quinoa, it showed that $H$. X1 gave the highest DPPH radical scavenging capacity and reducing power, F.G1 gave the highest superoxide anion radical scavenging ability. Either the starter strains or fermentation time resulted in a significant change in the antioxidant activities.

Keywords: Solid-state fermentation; Quinoa; Nutritional components; Antioxidant properties

\section{INTRODUCTION}

Quinoa (Chenopodium quinoa Willd.), an ancient pseudocereal, is originally cultivated in South America (Miranda et al., 2010). Compared with other cereals, quinoa has higher protein content, balanced amino acid composition, greater amounts of lysine and rich in dietary fiber, nutrients, vitamins, polyphenols and antioxidants properties (Ramos Diaz et al., 2017). This grain not only has high nutritional value, but also has shown positive results in preventing type-2 diabetes, osteoporosis, cardiovascular diseases, and autoimmune diseases (Antonio et al., 2010). So, the United Nations Food and Agriculture Organization (FAO) considered it as the only food originated from single plant that could prove all the basic nutrition for human health. With rich nutrition value and outstanding health function, quinoa can be widely used in food, daily chemical, agricultural and medicine industries (Antonio et al., 2010).

Quinoa has been traditionally used as supplement materials for making salads, porridges, granola bars, beer and bread (Bhargava et al., 2006; Tang et al., 2015). Quinoa also can be consumed as "healthy" snacks after processed by popping or extruding. What's more, this grain would be a good resource to provide soluble iron for human body (Koziol, 2010). However, neither cooking nor popping resulted in an effective utilization in the nutrition of quinoa. Finding an effective biological method to expand the traditional utilization of quinoa and to provide new, healthier and more nutritious food products of quinoa is urgent.

Solid-state fermentation (SSF), an older fermentation method, is fermented on solid substrates with little water or without free water (Yang et al., 2013). This biotechnological method has been traced back to 2000 years ago in China. The fermented products represent a rich source of nutrients, and have been preserved longer than other food process, such as steaming, parboiling, pan-frying or deep frying. This process has attracted more attention of functional foods manufactures for developing food supplements or materials (Abdullah et al., 2017; Xu et al., 2018). It had been reported that the nutritional components of substrates would be enhanced after fermented by higher fungi in SSF (Zhai et al., 2015). Higher fungi are abundant sources of

\footnotetext{
${ }^{*}$ Corresponding author:

Sheng Wan Zhang, School of Life Sciences, Shanxi University, Taiyuan, Shanxi Province, PRC. Tel: +86 0351-7639426, E-mail: zswan@ sxu.edu.cn;15364619285@163.com
}

Received: 12 November 2018; $\quad$ Accepted: 19 January 2019 
useful natural products with good biological activity. Many mushrooms have obtained interests as food or medicinal supplements (Xu et al., 2018). Agaricus bisporus is popularly known as the white button mushrooms, and has positive effects in treating cancer (Kimatu et al., 2017). Helvella lacunosa, grows naturally in North-West region of China, is well known as "Muer mushrooms" has been widely used for enhancing immunity and deeply loved by local people for its taste delicious (Zheng, 1987). Many reports indicated that the fungus is rich in polysaccharide, antioxidant properties and commonly used as food or medicine (Teng et al., 2013). Fomitiporia (Hymenochaetales, Basidiomycota), typified by Fomitiporia langloisii based on the morphological characters (Dai et al., 2008). The mushroom, also known as "Shushe" has been widely used for preventing many chronic diseases due to their superior antioxidant properties. The researches on Fomitiporia have receieved great attention because of their curative properties these years. It reported that the fungus is particularly rich in pregnenolone type triterpenes and exhibited positive effects on treating cancer (Ota et al., 2014; Chepkirui et al., 2018). In this way, the three fungi would be good candidates as materials for developing food or medicines. There are, however, the utilization of those fungus were limited owing to rare in nature and difficult to domestic cultivate. During SSF, the mycelia growth in the interface between the solid and aqueous phases, so the mycelia and substrate united firmly. Therefore, the fermented product combines the nutrients of the strain itself and the substrate. Thus, solid-state fermentation with edible mushrooms is a good manufacturing process to provide healthier and more nutritious food products.

In our paper, the object was to evaluate the effect on nutrients and antioxidants of quinoa fermented by three fungi in SSF. Because fermentation time is considered to be important influence factor with respect to the antioxidant activities of fermented products (Xu et al., 2018), the relationship between antioxidant properties and fermentation time was also analyzed.

\section{MATERIALS AND METHODS}

\section{Microorganisms}

Mycelia of AS2796 were obtained from Shanxi University, China. H.X1 and F.G1 were obtained from the culture collection at the Institute of Edible Fungi, Shanxi Academy of Agricultural Sciences, China. All the strains were subculture on potato agar dextrose (PDA) at $25^{\circ} \mathrm{C}$.

\section{Solid-state fermentation}

SSF was performed after inoculating fungi mycelia on quinoa grains ( 35 days). Briefly, the mixture of $180 \mathrm{~g}$ whole quinoa grains, $3 \mathrm{~g} \mathrm{CaCO}_{3}, 3 \mathrm{~g} \mathrm{CaSO}_{4}$ was soaked $12 \mathrm{~h}$ with
$170 \mathrm{~mL}$ tap water and sub-packed in $500 \mathrm{~mL}$ Canned Bottle, then sterilized at $121^{\circ} \mathrm{C}$ for $90 \mathrm{~min}$, cooled and inoculated. Inoculation was performed using the mycelia from PDA. Mixtures were incubated at $25^{\circ} \mathrm{C}$ in darkness. The test samples were obtained aseptically during fermentation $(0$, $7,14,21,28,35$ days) for analysis.

\section{Extracts preparation}

The ethanol extraction of all the three fungi were performed as explained in Xu et al. (2018). The test samples dried $\left(65^{\circ} \mathrm{C}\right)$ to constant weight, then individually milled and sieved. $3 \mathrm{~g}$ sample was extracted by $90 \mathrm{~mL}$ of $75 \%$ ethanol at $25^{\circ} \mathrm{C}$ for 24 hours and centrifuged by filtration. The resultant extracts were extracted with same volume of ethanol and evaporated at $40^{\circ} \mathrm{C}$ for $24 \mathrm{~h}$ in an incubator. The extracts were stored at $4^{\circ} \mathrm{C}$ without light.

\section{Main nutritional component assay}

The contents of total protein were determined by the Kjeldahl method (Chen and Li, 2014). The reducing sugar contents were determined by the 3, 5-2 nitro salicylic acid methods (DNS method) (Chen and Li, 2014). The crude fat was determined by the method of Soxhlet extraction (Chen and Li, 2014). The soluble starch was determined by the method of anthrone colorimetry (Chen and Li, 2014).

\section{Determination of the total phenol contents}

The TPCs were determined in the ethanolic extracts by Folin-Ciocalteu's reagent as explained previously (Xu et al., 2018). Briefly, each extract (100 uL) was dissolved in $100 \mathrm{uL}$ of $50 \%$ Folin-Ciocalteu's reagent. The mixture $(100 \mathrm{uL})$ was incubated at $25^{\circ} \mathrm{C}$ for 3 minutes and then added to $2 \mathrm{~mL}$ of $2 \%$ saturated $\mathrm{Na}_{2} \mathrm{CO}_{3}$. The final volume was made up to $5 \mathrm{~mL}$ with sterilized water. Then stand for 90 minutes without light. The absorbance was measured at $750 \mathrm{~nm}$. The total phenols was calculated on the basis of the calibration curve of gallic acid.

\section{Determination of the antioxidant properties}

Reducing power was determined by the method described as Kang et al. (2017). The absorbance was measured at $700 \mathrm{~nm}$.

Scavenging ability on DPPH (1,1-diphenyl-2-picrylhydrazyl) radicals was determined by the methods described by $\mathrm{Xu}$ et al. (2018). Each extracts $(2.0 \mathrm{~mL})$ were mixed with DPPH solution $(2.0 \mathrm{~mL}, \mathrm{v}: \mathrm{v}=1: 1)$ and $\mathrm{A} \mathrm{MeOH} / \mathrm{H}_{2} \mathrm{O}$ solution $(2.0 \mathrm{~mL}, 400 \mathrm{uM})$. Extraction for $30 \mathrm{~min}$ at $25^{\circ} \mathrm{C}$, the ability of the extracts to scavenge DPPH radical was assessed by measuring its absorbance at $517 \mathrm{~nm}$.

Scavenging ability on superoxide anion radicals was determined using the vitro blue tetrazolium (NBT) method (Chen and Li, 2014). 
The ferrous ion chelating ability of ethanolic extracts was determined as described by Zhai et al., (2015).

The $\mathrm{EC}_{50}$ values corresponded to the extract concentration by which $50 \%$ of maximum absorbance was reached, and it was determined by liner regression analysis.

\section{Experimentation and analysis}

All results were carried out in triplicates. Data in present study were subjected to one-way analysis of variance (ANOVA) at the level of 0.05. Experimental values are given as means \pm standard deviation (SD).

\section{RESULTS}

\section{Main nutritional components of fermented quinoa}

As shown in Table 1, the nutrient components of the fermentation products were analyzed. The content of starch in quinoa without fermentation is as high as $52.21 \%$, while the content of reducing sugar is only $1.02 \%$. After SSF with three fungi (35 days), the content of starch decreased to $18.46 \% \sim 44.83 \%$, while the content of reducing sugar was up to $2.64 \% \sim 5.62 \%$. The protein content of fermented quinoa also increased significantly, reaching $19.34 \% \sim 28.46 \%$, which were $159 \% \sim 234 \%$ higher than that of control. Crude fat content also decreased to $3.31 \% \sim 4.42 \%$ from $5.64 \%$ (unfermented quinoa).

\section{Total phenolic contents of fermented quinoa}

Phenolic compounds have strong antioxidant properties and play an important role in maintaining human health (Ullah et al., 2016) and they work well in DPPH radical scavenging, reducing agents, free radical scavenging, chelating metal and inhibiting lipid peroxidation (Tao et al. 2016; Gauthier et al., 2016). As shown in Fig. 1, either the fermentation time or the starter strains resulted in a significant change in the total phenol contents after SSF with AS2796, H.X1 and F. G1. Through ANOVA, the total phenolics of fermented quinoa had extremely significant correlation with the fermentation time $(R=0.935,0.911$ and 0.927 , respectively). After SSF (35 days), the total phenolics of fermented quinoa reached their maximum value: $1.38 \mathrm{mg} / \mathrm{g}, 1.30 \mathrm{mg} / \mathrm{g}$ and $1.33 \mathrm{mg} / \mathrm{g}$, which were $97 \%, 86 \%$ and $90 \%$ higher than that in unfermented quinoa, respectively. According to the enhancement rates, it was showed that quinoa fermented by AS2796 was significant higher than the other fungi $(P<0.05)$.

\section{Antioxidant properties of fermented quinoa}

The antioxidant properties assayed herein are summarized in Fig. $(2,3,4,5)$. The $\mathrm{EC}_{50}$ value was used to represent their effectiveness of antioxidant properties (Lee et al., 2016).

\section{Scavenging ability on DPPH radicals of fermented quinoa}

From Fig.2, the $\mathrm{EC}_{50}$ value of DPPH radicals scavenging ability in unfermented quinoa were $29.05 \mathrm{mg} / \mathrm{mL}$, after SSF with three strains (AS2796, H.X1 and F.G1), the lowest EC 50 values were obtained (35 days) $(0.11 \mathrm{mg} / \mathrm{mL}, 0.24 \mathrm{mg} / \mathrm{mL}$ and $0.17 \mathrm{mg} / \mathrm{mL}$, respectively). The correlation coefficient in quinoa between the scavenging ability and fermentation time were $-0.797,-799$ and -0.792 , respectively. The DPPH scavenging rate was ordered: strain $H$. X1 (92\%) $>$ AS2796 (88\%) > F.G1 (86\%).

\section{Reducing power in fermented quinoa}

The results of Fig.3 showed that the fermentation time has a negative effect on the reducing power $(R=-0.898,-0.904$ and -0.891 , respectively). After fermented 35 days, the $\mathrm{EC}_{50}$ reached its minimum value $(8.19 \mathrm{mg} / \mathrm{mL}, 8.32 \mathrm{mg} / \mathrm{mL}$ and $8.23 \mathrm{mg} / \mathrm{mL}$, respectively). Contrastly, the reducing rate was ordered: H. X1 (98\%)> AS2796 (92\%) > F. G1 (82\%).

\section{Superoxide anion radical scavenging ability in fermented quinoa}

From Fig.4, the superoxide anion radical scavenging ability in fermented quinoa had significant negative correlation with the fermentation time $(R=-0.980,-0.981$ and -0.982 , respectively). The lowest $\mathrm{EC}_{50}$ values of fermented quinoa were $9.15 \mathrm{mg} / \mathrm{mL}, 9.28 \mathrm{mg} / \mathrm{mL}$ and $9.18 \mathrm{mg} / \mathrm{mL}$, respectively, which were obtained after a fermentation of 35 days. The radical scavenging ability was ordered: F.G1 $(96 \%)>$ H.X1 $(93 \%)>$ AS2796 $(90 \%)$.

\section{Ferrousion chelating ability in fermented quinoa}

The data showed in Fig. 5 demonstrated that the ferrous ion chelating ability had negative correlation with the fermentation time $(R=-0.656,-0.657$ and -0.701 , respectively). The $\mathrm{EC}_{50}$ would reach its lowest after a fermentation of 35 days $(13.79 \mathrm{mg} / \mathrm{mL}, 13.62 \mathrm{mg} / \mathrm{mL}$ and $13.56 \mathrm{mg} / \mathrm{mL}$, respectively). The chelating rate was ordered: F. G1 $(94 \%)>$ H. X1 $(89 \%)>$ AS2796 (84\%).

Table 1: Main nutritional components of fermented quinoa

\begin{tabular}{lcccc} 
Sample & Total protein $(\mathbf{g} / \mathbf{1 0 0 g})$ & Crude fat $(\mathbf{g} / \mathbf{1 0 0 g})$ & Soluble starch $(\mathbf{g} / \mathbf{1 0 0 g})$ & Reducing sugar $(\mathbf{g} / \mathbf{1 0 0 g})$ \\
\hline Unfermented & $12.18 \pm 0.52 \mathrm{c}$ & $5.64 \pm 0.94 \mathrm{~d}$ & $52.21 \pm 0.15 \mathrm{a}$ & $1.02 \pm 0.03 \mathrm{~b}$ \\
AS2796 & $28.46 \pm 1.23 \mathrm{a}$ & $4.38 \pm 0.06 \mathrm{c}$ & $44.83 \pm 1.03 \mathrm{~b}$ & $3.13 \pm 0.13 \mathrm{~b}$ \\
H. lacunosa X1 & $23.14 \pm 1.31 \mathrm{~b}$ & $4.42 \pm 0.14 \mathrm{a}$ & $39.33 \pm 0.14 \mathrm{~b}$ & $5.62 \pm 0.01 \mathrm{a}$ \\
F. yanbeiensis G1 & $19.34 \pm 0.23 \mathrm{c}$ & $3.31 \pm 0.27 \mathrm{~b}$ & $18.46 \pm 1.08 \mathrm{c}$ & $2.64 \pm 0.08 \mathrm{~b}$ \\
$P$ value & 0.002 & 0.438 & 0.113 & 0.016 \\
\hline
\end{tabular}




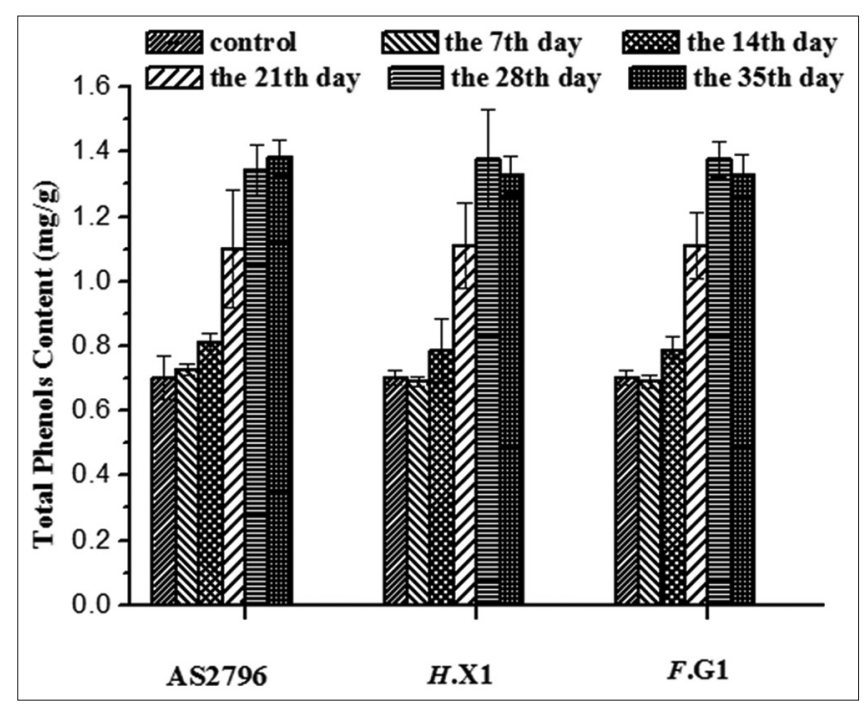

Fig1. Effects of fermentation time on total phenol content of fermented quinoa.

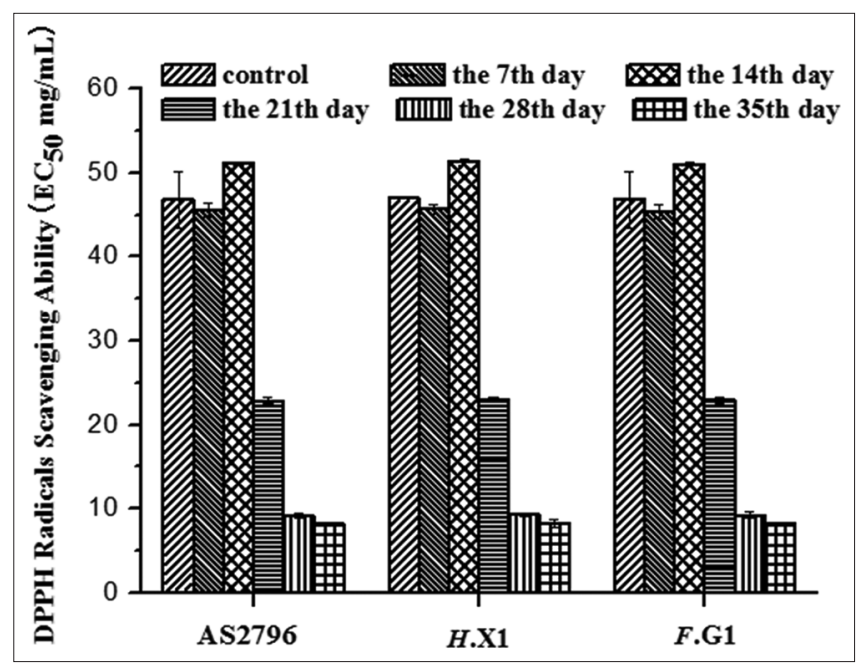

Fig 2. Effects of fermentation time on scavenging ability on DPPH radicals of fermented quinoa.

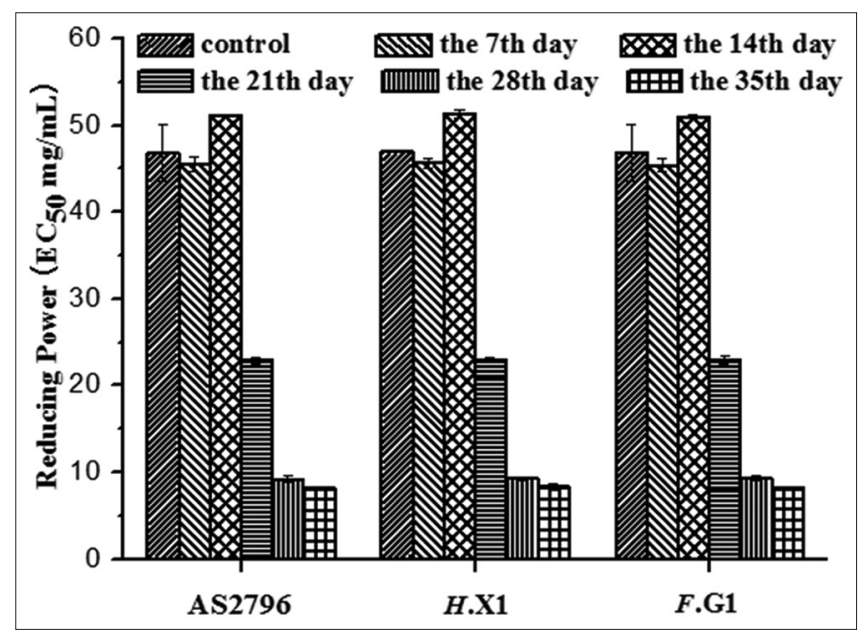

Fig 3. Effects of fermentation time on reducing power in fermented quinoa.

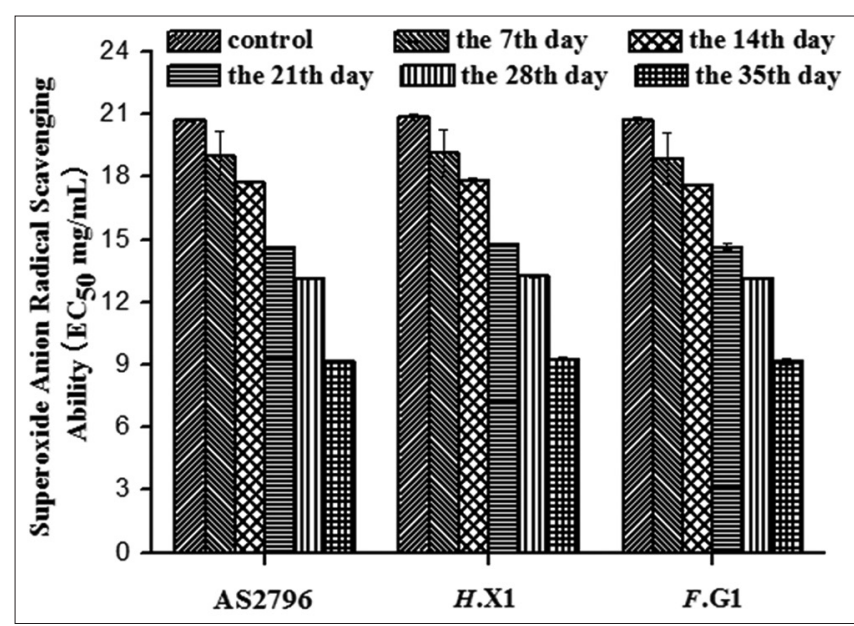

Fig 4. Effects of fermentation time on superoxide anion radical scavenging ability in fermented quinoa.

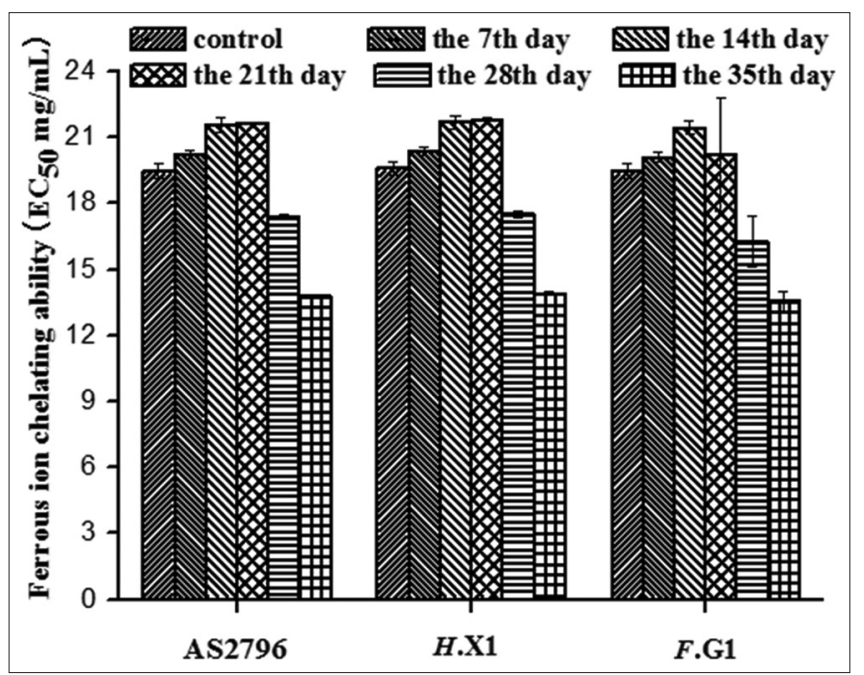

Fig 5. Effects of fermentation time on ferrous ion chelating ability in fermented quinoa.

Fig. $(2,3,4,5)$ showed that the $\mathrm{EC}_{50}$ values in the four different assays of antioxidant ability had negative correlation with fermentation time, which meant that the antioxidant ability enhanced allowing with the fermentation time.

\section{DISCUSSIONS}

From all the results, it obtained that the change rate of different nutritional components in fermented quinoa varied with the starter fungi. For AS2796, the total protein content was highest $(28.46 \mathrm{~g} / 100 \mathrm{~g})$, while the highest value of reducing sugar was obtained by $H$.X1. Experiments of Kang et al. (2017) and Zhai et al. (2015) indicated that both the starter fungi and fermented grains in SSF could have an impact on the nutrition of the fermented products, and this depended on the starch structure of 
cereals and the metabolic mechanism of fungi. It showed that higher fungus has the ability to improve the nutritional components, and the improved rates accordance with the starter strains. Additionally, the comparative study in nutritional components of fermented - quinoa showed that the reducing sugar contents of fermented quinoa were increased while the starch contents were reduced. At this point, no satisfactory explanation can be offered. It is likely that some starch was hydrolyzed to sugar. These experiments also implied that the three medicinal and edible mushrooms have the ability to produce $\alpha$-amylase in fermentation on quinoa. Zhai et al. (2015) reported that Agaricus blazei $i$ could produce $\alpha$-amylase in SSF on grains. In present study, the degrade rate of starch was different due to the fermented fungi. This result could be explained that each fungus has its own metabolism and the bioactive compounds could be modified in SSF by fungi (Dulf et al., 2016; Liu et al., 2017; Okeke et al., 2015). Thus we should select different starter strain for SSF on quinoa according to different fermentation purpose. Some reports also reported the fermentation could enhance the nutrition value of quinoa by promoting the quinoa release small peptides and amino acids (Dallagnol et al., 2013). In this case, it is necessary to determine the amylase and nutritious contents of the fermented products by different fungus to explain all these results.

Mushrooms could generate a variety of secondary metabolites, such as polyphenols and polysaccharose in vivo or vitro, which have been shown strong antioxidant activity (Fardet and Christian, 2008). The polyphenols act as antioxidants due to the redox properties (Han et al., 2005; Rasane et al. 2015; Agu et al., 2015). However, there was an exception in our experiment. The total phenol content of fermented quinoa on the $28^{\text {th }}$ day with $F$. G1 was reached the highest value $(1.38 \mathrm{mg} / \mathrm{g})$, while the lowest $\mathrm{EC}_{50}$ of the antioxidant properties (i.e. DPPH radical scavenging capacity, reducing power, ferrous ion chelating ability and superoxide anion radical scavenging ability) was obtained on the $35^{\text {th }}$ day. Many reports showed that quinoa is rich in minerals, vitamins, and contains a variety of antioxidant properties, such as polyphenols, phytosterols, and flavonoids. At this point, the results could be explained that polyphenols are not only antioxidants in the fermentation quinoa, but also other bioactive substances. A large number of metabolites such as ergotoxine, flavonoids, anthocyanin, vitamin $\mathrm{C}$ and $\mathrm{E}$ in the fermentation process may improve the contents of antioxidant properties of the fermented products (Zhai et al., 2015; Bei et al., 2018; Xu et al., 2018; Cai et al., 2012). In further research, evaluate the relationship between bioactive compounds and antioxidant activity of the fermented quinoa would be the focus, and the chemical compounds in fermented quinoa should be isolated and purified separately.
There are many assays to evaluate the antioxidant activity of raw and processed cereal grains. Results showed that the antioxidant capacity values are varied with the kind of assay used, the oxidation substrate and the extraction method. In this study, the antioxidant activity was determined through four different assays. The results showed that the antioxidant properties of fermented quinoa improved compared with the control and varied with the fermentation time. Our results agreed with the research of Zhai et al. (2015), who evaluated the antioxidant capacity of grains and fermented grains, and found that fermented cereals had higher antioxidant activity compared with raw ones. Products based on SSF process with fungi are relatively high in antioxidantive property, which attributed to the characteristics of starter organisms and the presence of antioxidant components of solid substrates, including the polyphenolic compounds (Han et al., 2005). In our study, $H$. X1 gave the highest DPPH radical scavenging capacity and reducing power, while F.G1 gave the highest superoxide anion radical scavenging ability. Either the starter strains or fermentation time resulted in a significant change in the antioxidant activities. Why is that? H.X1 belongs to ascomycetes, while F.G1 and AS2796 belong to basidiomycetes. Perhaps the enhanced rate of antioxidant properties on fermented quinoa was depended on the physiology of starter fungi. Additionally, antioxidant property of foods can be enhanced during thermal treatment because of the formation of antioxidant non phenolic compounds such as Maillard products, which have been related to increased free radical scavenging properties (Ullah et al., 2016). Recently, fermented foods have attracted increased interest from nutritionists, food manufacturers and the general public. In our research, we analyzed and identified fermented quinoa with high TPC and antioxidantive capacity by the single fungi in SSF. So, no attempt was made to analyze the mixed strains. In further research, the TPC and antioxidantive capacity of quinoa fermented by mixed strains would be evaluated in order to provide new and more nutritious foods.

Quinoa is an alternative cereal for vegetarians and vegans, because it is free of lactose and cholesterol and also has ability to suffering from coeliac disease and gluten-allergy problems (Nowak et al., 2016). The cereal has been cultivated over 30 years in Shanxi Province, the seed of quinoa can be milled into flour and used for bread-making, pasta, biscuits, and other processed foods. In the present study, the quinoa fermented by F.G1 and H.X1 exhibited strong antioxidant activity than unfermented quinoa. To meet people's increasing demand, the quinoa-fungi products can be alternative to natural healthier diets. 


\section{ACKNOWLEDGEMENTS}

This research was supported by institutional funds from the Shanxi Academy of Agricultural Sciences (2017zx-20). The authors wish to thank Prof. Shang Guo of Shanxi Academy of Agricultural Sciences, Taiyuan, China, who kindly provided the financial support for this study.

\section{Authors' contributions}

The Prof. Shengwan Zhang designed the experiments. Lina Xu conducted the experiments and analyzed the data. Prof. Shang Guo provided the financial support for this study.

\section{CONFLICT OF INTEREST}

None declared.

\section{REFERENCES}

Antonio, V., M. Margarita, V. Judith, U. Elsa, P. Luis and M. Enriquea. 2010. Nutrition facts and functional potential of quinoa (Chenopodium quinoa willd), an ancient Andean grain: A review. J. Sci. Food Agric. 90: 2541-2547.

Agu, R. C., T. A. Bringhurst and J. M. Brosnan. 2015. Production of grain whisky and ethanol from wheat, maize and other cereals. J. Inst. Brewing. 112: 314-323.

Abdullah, N., R. Abdulghani, S. M. Ismail and M. H. Z. Abidin. 2017. Immune-stimulatory potential of hot water extracts of selected edible mushrooms. Food Agric. Immunol. 28: 374-387.

Bei, Q., G. Chen, F. Lu, S. Wu and Z. Wu. 2018. Enzymatic action mechanism of phenolic mobilization in oats (Avena sativa L.) During solid-state fermentation with monascus anka. Food Chem. 245: 297-304.

Bhargava, A., S. Shukla and D. Ohri. 2006. Chenopodium quinoa--an Indian perspective. Ind. Crops Prod. 23: 73-87.

Cai, S., O. Wang, W. Wu, S. Zhu, F. Zhou and B. Ji. 2012. Comparative study of the effects of solid-state fermentation with three filamentous fungi on the total phenolics content (tpc), flavonoids, and antioxidant activities of subfractions from oats. J. Agric. Food Chem. 60: 507-513.

Chen, J. H. and J. Li. 2014. Biochemistry Experiment, $5^{\text {th }}$ ed. Science Press, Beijing.

Chepkirui, C., W. C. Sum, T. Cheng, J. C. Matasyoh, C. Decock and M. Stadler. 2018. Aethiopinolones a-e, new pregnenolone type steroids from the East African basidiomycete fomitiporia aethiopica. Molecules. 23: E369.

Dallagnol, A. M., M. Pescuma, G. F. De Valdez and N. Rollã. 2013. Fermentation of quinoa and wheat slurries by Lactobacillus plantarum Crl 778: Proteolytic activity. Appl. Microbiol. Biotechnol. 97: 3129-3140.

Dulf, F. V., D. C. Vodnar and C. Socaciu. 2016. Effects of solid-state fermentation with two filamentous fungi on the total phenolic contents, flavonoids, antioxidant activities and lipid fractions of plum fruit by-products. Food Chem. 209: 27-36.

Dai, Y. C., B. K. Cui and D. Cony. 2008. A new species of Fomitiporia (hymenochaetaceae, basidiomycota) from china based on morphological and molecular characters. Mycolog. Res. 112: 375-380.
Fardet, A. E. and R. Christian. 2008. Is the in vitro antioxidant potential of whole-grain cereals and cereal products well reflected in vivo? Cereal Sci. 48: 258-276.

Gauthier, L., M. N. Bonnin-Verdal, G. Marchegay, L. Pinson-Gadais, C. Ducos and F. Richard-Forget. 2016. Fungal biotransformation of chlorogenic and caffeic acids by Fusarium graminearum: New insights in the contribution of phenolic acids to resistance to deoxynivalenol accumulation in cereals. Int. J. Food Microbiol. 221: $61-68$.

Han, J. R., C. H. An and J. M. Yuan. 2005. Solid-state fermentation of cornmeal with the basidiomycete Ganoderma lucidum for degrading starch and upgrading nutritional value. J. Appl. Microbiol. 99: 910-915.

Kang, M., F. H. Zhai, X. X. Li and J. R. Han. 2017. Total phenolic contents and antioxidant properties of buckwheat fermented by three strains of Agaricus. Cereal Sci. 73: 138-142.

Koziol, M. J. 2010. Afrosimetric estimation of threshold saponin concentration for bitterness in quinoa (Chenopodium quinoa Willd). J. Sci. Food Agric. 54: 211-219.

Kimatu, B. M., L. Zhao, B. Yuan, G. Ma, W. Yang and F. Pei. 2017. Antioxidant potential of edible mushroom (Agaricus bisporus) protein hydrolysates and their ultrafiltration fractions. Food Chem. 230: 58-67.

Liu, J., J. Chen, Y. Fan, X. Huang and B. Han. 2017. Biochemical characterization and dominance of different hydrolases in different types of daqua Chinese industrial fermentation starter. J. Sci. Food Agric. 98: 113-121.

Lee, L. S., E. J. Choi, C. H. Kim, J. M. Sung, Y. B. Kim and D. H. Seo. 2016. Contribution of flavonoids to the antioxidant properties of common and tartary buckwheat. J. Cereal Sci. 68: 181-186.

Miranda, M., A. Vegagálvez, J. López, G. Parada, M. Sanders and M. Aranda. 2010. Impact of air-drying temperature on nutritional properties, total phenolic content and antioxidant capacity of quinoa seeds (Chenopodium quinoa willd.). Ind. Crops Prod. 32: 258-263.

Nowak, V., J. Du and U. R. Charrondière. 2016. Assessment of the nutritional composition of quinoa (Chenopodium quinoa willd). Food Chem. 193: 47-54

Okeke, C. A., C. N. Ezekiel, C. C. Nwangburuka, M. Sulyok, C. O. Ezeamagu and R. A. Adeleke. 2015. Bacterial diversityand mycotoxin reduction during maize fermentation (steeping) for ogi production. Front. Microbiol. 6: 140.

Ota, Y., T. Hattori, H. Nakamura, Y. Terashima, S. S. Lee and Y. Miyuki. 2014. Taxonomy and phylogenetic position of Fomitiporia torreyae, a causal agent of trunk rot on sanbu-sugi, a cultivar of Japanese cedar (Cryptomeria japonica) in Japan. Mycologia. 106: 66-76.

Rasane, P., A. Jha, A. Kumar and N. Sharma. 2015. Reduction in phytic acid content and enhancement of antioxidant properties of nutricereals by processing for developing a fermented baby food. J. Food Sci. Technol. 52: 1-16.

Ramos, D. J. M., L. Sundarrajan, S. Kariluoto, A. M. Lampi, S. Tenitz and K. Jouppila. 2017. Effect of extrusion cooking on physical properties and chemical composition of cornâ based snacks containing amaranth and quinoa: Application of partial least squares regression. J. Food Process Eng. 40: 118-120.

Tao, Y. Y., X. C. Wei and J. X. Bao. 2016. From rice bag to table: Fate of phenolic chemical compositions and antioxidant activities in waxy and non-waxy black rice during home cooking. Food Chem. 191: 81-90.

Teng, L. P., H. Zeng and H. B. Wang. 2013. Study on the deproteinization technology of crude polysaccharide from Helvella leucopus. J. Henan Agric. Sci. 42: 138-137.

Tang, Y., X. Li, B. Zhang, P. X. Chen, R. Liu and R. Tsao. 2015 
Characterisation of phenolics, betanins and antioxidant activities in seeds of three Chenopodium quinoa Willd. Genotypes. Food Chem. 166: 380-388.

Ullah, F., M. Ayaz, A. Sadiq, A. Hussain, S. Ahmad and M. Imran. 2016. Phenolic, flavonoid contents, anticholinesterase and antioxidant evaluation of iris Germanica Var; Florentina. Natl. Prod. Res. 30: 1440-1444.

Xu, L. N., S. Guo and S. W. Zhang. 2018. Effects of solid-state fermentation with three higher fungi on the total phenol contents and antioxidant properties of diverse cereal grains. FEMS
Microbiol. Lett. 11: 132-140.

Yang, F. C., Y. H. Yang and H. C. Lu. 2013. Enhanced antioxidant and antitumor activities of Antrodia cinnamomea, cultured with cereal substrates in solid state fermentation. Biochem. Eng. J. 78: 108-113.

Zhai, F. H., Q. Wang and J. R. Han. 2015. Nutritional components and antioxidant properties of seven kinds of cereals fermented by the basidiomycete Agaricus blazei. Cereal Sci. 65: 202-208.

Zheng, Q. Z. 1987. A delicious fungi needed to be developed urgentlythe research of Helvella lacunosa. Edible fungi. 1. 